Original Paper http://ajol.info/index.php/ijbcs http://indexmedicus.afro.who.int

\title{
Changements environnementaux et vulnérabilité des écosystèmes dans le bassin-versant béninois du fleuve Niger
}

\author{
E. AMOUSSOU ${ }^{1,2 *}$, S. H. TOTIN VODOUNON ${ }^{1,2}$, A. HOUGNI ${ }^{3}$, E. W. VISSIN ${ }^{2}$, \\ C. HOUNDENOU ${ }^{2}$, G. $\mathrm{MAHE}^{4}$ et M. BOKO ${ }^{2}$ \\ ${ }^{1}$ Département de Géographie et Aménagement du Territoire, Université de Parakou, BP 123 Parakou, Bénin. \\ ${ }^{2}$ Laboratoire Pierre PAGNEY, Climat, Eau, Ecosystème et Développement (LACEEDE), Université \\ d'Abomey-Calavi, République du Bénin, 03 BP1122 Cotonou, Bénin. \\ ${ }^{3}$ Institut National de Recherches Agronomiques du Bénin (INRAB), Direction de Recherche Coton et fibres, \\ Bénin. \\ ${ }^{4}$ Institut de Recherche pour le Développement (IRD), Laboratoire HydroSciences Montpellier (HSM), \\ Université de Montpellier 2, France. \\ *Auteur correspondant; E-mail : ernestamoussou@gmail.com
}

\section{RESUME}

Les régions soudano-sahéliennes sont confrontées aux défis de l'explosion démographique et des changements climatiques qui induisent des modifications inévitables dans les systèmes de gestion des terres. Les processus naturels et humains ont des conséquences sur les capacités des systèmes de production. Ce phénomène conduit dans plusieurs régions à la dégradation des ressources naturelles, à la faible productivité des terres et à la perte de la biodiversité comme c'est le cas déjà dans le bassin du fleuve Niger au Bénin. L'analyse s'appuie sur les données climatologiques de 1951-2010, les statistiques de production agricole de Banikoara de 1978 à 2010. Ces données sont complétées par celles des enquêtes de terrain et des résultats d'une séance de travail avec les acteurs de gestion des ressources naturelles dans le bassin. La rareté croissante des ressources naturelles et l'augmentation des besoins ont entraîné une forte compétition et divers conflits entre les utilisateurs du bassin. En effet, les stratégies les plus envisageables sont : la culture de contre-saison dans les bas-fonds, la réduction des superficies consacrées à la culture du coton, l'utilisation des plantes vénéneuses pour droguer les poissons, le reboisement en amont des retenues d'eau et sur les berges.

(C) 2016 International Formulae Group. All rights reserved.

Mots clés : Bassin béninois, Niger, écosystèmes sensibles, changements environnementaux, gestion.

\section{Environmental change and ecosystems vulnerability on Benin Catchment of the River Niger}

\begin{abstract}
Sahel regions are facing population rapid growth and climate change challenges leading to inevitable modification of land management system. These natural and human processes have an impact on production systems capacity. In several regions, this phenomenon lead to degradation of natural resources, reduction of
\end{abstract}


land productivity and loss of biodiversity as already experienced on Benin Basin of the River Niger. Analysis is based on climate data over the period 1951-2010 and crop production data of the Municipality of Banikoara from 1978 to 2010. Field works and results of focus group with users and the basin natural resource managers were complementary methods. Increased scarcity of natural resources and of the needs have result on strong competition and various conflicts between natural resources users on the basin. Indeed, possible strategies are: dry season crop production on the wetland, reduction of cotton cultivation area, poisonous plants use for drugging fish, reforestation of upstream of the small reservoir and River bank.

(c) 2016 International Formulae Group. All rights reserved.

Keywords: Benin, Niger River basin, ecosystem management, environmental changes.

\section{INTRODUCTION}

L'absence, la rareté, l'excès ou la mauvaise répartition spatio-temporelle des pluies peuvent être générateurs de crises climatiques et économiques et très souvent aussi, d'instabilité sociale et politique (GIEC, 2007 ; Totin, 2010; Yabi et Afouda, 2012; Gbaguidi et al., 2015). Sur ce plan, l'Afrique de l'ouest, où la plupart des pays ont une économie fondée sur le secteur primaire, en particulier sur l'agriculture pluviale, apparaît comme une région particulièrement sensible (GIEC, 2007 ; Vissin, 2007). La baisse très marquée de la pluviométrie observée dans cette région au cours des décennies 1970 et 1980 a eu des répercussions importantes, aux plans hydrologique et agronomique, mais aussi économique et social (Amoussou, 2010 ; Katé et al., 2016). Ainsi, la baisse des rendements des cultures vivrières et commerciales dans le bassin-versant du Niger au Bénin entraîne une utilisation non contrôlée et intensive des intrants agricoles. La conséquence directe est une dégradation des écosystèmes et une pollution des eaux suite au ruissellement et au lessivage des sols.

Cette étude a pour objectif d'analyser le degré de sensibilité des écosystèmes (les eaux et les terres) et les stratégies de gestion dans un contexte de climat et de l'environnement modifiés dans le bassin du fleuve Niger au Bénin. Ce travail s'appuie sur des données climatologiques (pluie), agronomiques (production, superficies emblavées et rendement), des résultats issus du stakeholder de Kandi lors du lancement du projet Esprit en octobre 2009 et des observations de terrain.
Le bassin béninois du fleuve Niger est situé à l'extrême nord-est du Bénin (Figure 1). Il couvre une superficie d'environ $48000 \mathrm{~km}^{2}$, soit $42 \%$ de la superficie totale du Bénin $\left(114763 \mathrm{~km}^{2}\right)$. Situé entre les latitudes $10^{\circ}$ et $12^{\circ} 30^{\prime}$ Nord et les longitudes $1^{\circ} 32^{\prime}$ et $3^{\circ} 50^{\prime}$ Est, il regroupe les sous bassins de la Mékrou, de l'Alibori et de la Sota, et est orienté en général SSW-NNE.

Le régime pluviométrique est de type tropical unimodal avec un maximum marqué en août. Le potentiel précipitable disponible dépend en grande partie de l'apport hydrique de la mousson atlantique et caractérisé par deux saisons contrastées : une saison sèche et une saison pluvieuse. L'espace géographique du bassin est caractérisé par une concentration des pluies (96\% annuels moyens) sur six mois (mai-octobre) (Vissin, 2007), à l'image de la disposition saisonnière des pluies en pays soudano-sahéliens.

Le bassin-versant est constitué de deux unités topographiques : la pénéplaine cristalline et le plateau de grès de Kandi. Les sols des bassins du Nord-Bénin résultent essentiellement de processus de ferrallitisation et de ferruginisation et s'étend sur les formations de socle à l'ouest et au sud et les grès de Kandi au nord-est (Vissin, 2007; Ouorou Barrè, 2014). Cette étude avait pour objectif l'évaluation des changements environnementaux et vulnérabilité des écosystèmes dans le bassin-versant béninois du fleuve Niger.

\section{MATERIEL ET METHODES}

Les données utilisées sont les précipitations mensuelles de 1951 à 2010 d'un 
réseau de neuf stations réparties dans le bassin et gérées par l'Agence pour la Sécurité de la Navigation Aérienne en Afrique et à Madagascar (ASECNA) de Cotonou (Bénin). Les analyses ont aussi porté sur les statistiques de production, de superficies emblavées et de rendements des principales cultures (coton, maïs, sorgho et igname) à Banikoara de 1978 à 2010. Elles sont recueillies au Ministère de l'Agriculture, de l'Elevage et de la Pêche (MAEP) et sont complétées par celles recueillies à Kandi lors du démarrage du projet Esprit. Pour ce faire, un questionnaire a été adressé à un groupe de 32 personnes de plusieurs catégories socioprofessionnelles (agriculteurs, éleveurs, pêcheurs, forestiers, Coordonnateurs de projet, $\mathrm{ONG}$ ) et une grille d'observations directes de terrain a été utilisée.

Des investigations ont été aussi réalisées par l'approche MARP (Méthode Accélérée de Recherche Participative) comportant des entretiens semi-directifs, des interviews et des focus-groups. Cette méthode a permis aux populations rurales et urbaines de présenter leurs conditions de vie en relation à la variation climatique et aux modifications environnementales dans le bassin béninois du fleuve Niger.

Détermination du champ de pluie du bassin du fleuve Niger au Bénin

Le champ pluviométrique a été déterminé pour mieux appréhender l'évolution spatiale des pluies dans le bassin. La méthode utilisée est celle d'interpolation cubique spline axée sur quatre points déjà utilisées par d'autres chercheurs (Oettli et Camberlin, 2005; Oettli, 2008) :

- Une régression linéaire multiple, en fonction de trois paramètres que sont la latitude, la longitude et l'altitude. Ainsi, l'estimation de la moyenne par interpolation sur l'ensemble du bassin, à partir de 720 (720=60 ans x 12 mois) mesures ponctuelles des neufs stations retenues et s'avère nécessaire. C'est une généralisation directe à $\mathrm{p}$ variables explicatifs de la régression linéaire simples. Elle permet de connaître la distribution de chaque variable prédictive à la variable à expliquer en neutralisant l'effet simultané de toutes les autres variables prédictives. Elle consiste ainsi, à estimer, à partir des données expérimentales (xi, yi) $(\mathrm{i}=1,2, \ldots . \mathrm{N})$, les paramètres $a_{0}$ et $a_{1}$ d'une loi liant les valeurs d'une variable aléatoire y à une variable indépendante non aléatoire $\mathrm{x}$, selon le modèle :

$$
y=E[y]+\varepsilon=a_{0} a_{1} x+\varepsilon
$$

où $\mathrm{E}[\mathrm{y}]$ désigne l'espérance mathématique de la variable aléatoire "y" et $\varepsilon$ est une variable aléatoire de moyenne nulle. La moyenne de la précipitation dans le bassin correspondant à la variable indépendante $\mathrm{x}$ est donnée par la loi linéaire $a_{0}+a_{1}{ }^{\mathrm{xi}}$. Le paramètre $a_{1}$ mesurant la sensibilité de E[y] à x, est appelé le coefficient de régression. Ainsi, ce modèle exprime la relation linéaire entre plusieurs facteurs qualitatifs que sont: la latitude, la longitude et l'altitude (Oettli, 2008; Amoussou, 2010). Le choix de ces trois variables permet de définir leur influence dans la répartition pluviale du bassin-versant. Cette précipitation estimée en tout point du bassin est ainsi définie à partir de la grille MNT (Modèle Numérique de Terrain) de SRTM30 (Shuttle Radar Topography Mission) (Gorokhovich et Voustianouk, 2006) qui est une mise à jour du format GTOPO30. Dans le cadre de cette étude, la grille est de $1 \mathrm{~km} \times 1$ $\mathrm{km}$. Après la régression linéaire multiple, les ratios obtenus sont interpolés.

- Une interpolation simple des ratios (rapport des pluies observées et de la pluie estimée par le modèle de régression) regroupe l'interpolation spatiale et l'interpolation cubique. Il s'agit d'une interpolation cubique des champs moyens de pluie d'une part, et de la variation interannuelle de précipitations c'est-à-dire des écarts de pluie à la moyenne d'autre part. Les ratios obtenus décrivent la répartition de la pluie indépendamment des coordonnées géographiques. Ils ne représentent qu'une fraction de la variabilité spatiale de la pluie. Cette interpolation cubique s'effectue, en ajoutant une surface, souvent un polynôme, dans chaque triangle. 
Les valeurs régionalisées dans les stations pluviométriques du champ, formant les sommets du grand triangle dans lequel sont issus les petits triangles, sont incluses pour l'interpolation. A cet effet, le poids de chacune de ces valeurs est égal à la portion de surface du grand triangle occupé par le petit triangle opposé au site.

- Ensuite, il est procédé à l'estimation pluviométrique moyenne et interannuelle qui est la reconstitution finale des champs de pluie $\mathrm{du}$ bassin. Elle fait intervenir la liaison entre les ratios ou résidus interpolés, complétée par les pluies estimées par le modèle de régression linéaire en prenant en compte les liaisons pluviométrie/topographie.

- Enfin, une validation croisée des précipitations estimées. Cette pratique consiste à valider l'estimation et le voisinage utilisé pour l'interpolation. Elle permet aussi de comparer la qualité prédictive des modèles sur la base d'un critère d'erreur (Philippon, 2002).

Pour mieux cerner l'évolution du climat dans le bassin, la carte de péjoration pluviométrique entre les deux normales (1951-1980 et 1981-2010) de trente chacune a été réalisée pour apprécier les secteurs les plus vulnérables à la baisse des précipitations. En outre, l'évolution des précipitations entre 1951-2010 passe également par la recherche des ruptures de stationnarité. De même les résultats des travaux de Vissin (2007) sur la dynamique du couvert végétal sont utilisés pour montrer les changements observés dans le bassin sur les unités d'occupation du sol entre les années 1979 et 1992 afin d'en déduire leurs impacts sur les écosystèmes.

\section{Recherche de rupture de stationnarité avec le test de Pettitt}

Le test de Pettitt par sa robustesse à détecter une rupture dans les séries chronologiques a été utilisé pour l'étude des modifications intervenues dans les séries pluviométriques (Servat et al., 1999 ; Totin et al., 2009). L'absence de rupture dans une série (Xi) de taille $\mathrm{N}$ constitue l'hypothèse nulle H0. La mise en œuvre de ce test suppose que pour tout instant $t$ compris entre 1 et $\mathrm{N}$, les séries chronologiques $x i, i=1, t$ et $x j, j=t+1, N$ appartiennent à la même population. La variable à tester est le maximum en valeur absolue de la variable Ut, $\mathrm{N}$ définie par :

$$
\begin{aligned}
& U_{t, N}=\sum_{i=1}^{t} \sum_{j=t+1}^{N} D_{i j} \\
& \text { Où } D_{i j}=\operatorname{sgn}\left(X_{i}-X_{j}\right) \text { avec } \operatorname{sgn}(x)=1 \\
& \text { si } x>0,0 \text { si } x=0 \text { et }-1 \text { si } x<0
\end{aligned}
$$

A partir de la théorie des rangs, Pettitt montre que si $\mathrm{k}$ désigne la valeur $\mathrm{KN}$ prise sur la série étudiée, sous l'hypothèse nulle, la probabilité de dépassement de la valeur k est exprimée approximativement par :

$\operatorname{Pr} o b\left(K_{N}>k \approx 2 \exp \left(-6 k^{2} /\left(N^{3}+N^{2}\right)\right)\right.$

$\mathrm{H} 0$ est rejetée pour un risque $\alpha$ de première espèce donnée, si la probabilité de dépassement estimée est inférieure à $\alpha$. La série comporte alors une rupture localisée au moment où est observé max $|\mathrm{Ut}, \mathrm{N}| \mathrm{t}=1, \mathrm{~N}-1$.

Elaboration de la base de règles de vulnérabilité

Pour une évaluation de la vulnérabilité, la base de règle occupe une place stratégique, car c'est elle qui renferme toute la logique nécessaire à l'établissement des liens entre les paramètres et les éléments de la base de faits comme l'illustre la Figure 2. De l'application de ces règles, va découler tout un raisonnement qui va aboutir à la caractérisation de la vulnérabilité face aux conséquences des changements environnementaux. La méthodologie de la vulnérabilité utilisée, est celle déjà testée par Olivry (2012) pour l'analyse de la vulnérabilité des écosystèmes dans un contexte de changements climatiques.

Pour mieux décrire et quantifier les problèmes auxquels sont confrontés les écosystèmes du bassin, le modèle DPSIR (Force motrice-Pression-État-ImpactRéponse), a servi de base à l'analyse des résultats issus des travaux de terrain, des traitements statistiques et cartographiques.

\section{RESULTATS}

Evolution interannuelle des précipitations dans le bassin

La Figure 3 présente l'évolution spatiale des précipitations dans le secteur 
d'étude. Elle montre une diminution des précipitations d'Est vers l'Ouest. C'est la région de Bembèrèkè et de Kérou voire Banikora qui reçoit les plus importantes quantités d'eau pluviale (900 à $1200 \mathrm{~mm}$ ). Ces communes sont situées à la limite du domaine tropical humide et bénéficient des atouts climatiques de ce domaine. Par contre, les autres régions du secteur d'étude sont sous l'influence du climat tropical sec (moins de $900 \mathrm{~mm}$ de pluie par an).

L'étude de la variabilité interannuelle présentée sur la Figure 4 indique une tendance significative à la baisse des précipitations de 1951 à 2010. Cette baisse est plus marquée dans les décennies 1970 et 1980 (cadre rouge sur la Figure 3) suivie d'une légère reprise au cours de la décennie 1990, moins humide que les décennies 1950 et 1960.

Pour mieux apprécier cette tendance évolutive, une analyse de la rupture de stationnarité observée dans la série est faite.

\section{Rupture de stationnarité dans les séries du champ pluviométrique du bassin}

La Figure 5 qui présente l'évolution de la variable $\mathrm{U}$ du test de Pettitt appliquée au champ de pluies du bassin béninois du fleuve Niger montre une baisse significative des lames d'eau précipitées depuis les années 1970. Cette rupture de stationnarité en 1969 significative à 99\%, confirment les résultats de Houndénou (1999) et Vissin (2007) qui ont trouvé également ces mêmes ruptures avec le test de Mann-Kendall sur la période 19501992.

Pour mieux cerner cette modification pluviométrique, une étude comparative du régime pluviométrique sur les deux souspériodes (1950-1969 et 1970-2010) est réalisée.

L'étude comparée des deux souspériodes permet de mettre en évidence la baisse marquée des hauteurs de pluie saisonnière sur la sous-période 1970-2010 de 12\% (soit environ $134 \mathrm{~mm}$ ). La Figure 6 montre aussi que les mois humides (juin à septembre) ont connu une baisse des précipitations. Il ressort de l'analyse de cette figure que ces mois qui totalisaient annuellement les $880 \mathrm{~mm}$ entre 1951-1969, enregistrent les $769 \mathrm{~mm}$ sur la sous-période 1970-2010 soit une baisse de 13\%. De même, les mois secs ont connu une baisse de $10 \%$, soit $23 \mathrm{~mm}$ de pluie entre les deux souspériodes. Cette baisse de la précipitation dans un environnement où la demande évaporatoire est élevée, un assèchement rapide et une baisse considérable de la disponibilité en eau s'observent.

La sous-période 1971-2010 est nettement déficitaire comme l'illustre la Figure 7, qui présente l'évolution spatiale des lames d'eau précipitée suivant les deux souspériodes de rupture. Les hauteurs de pluie annuelles ont complètement diminué sur la sous-période comparativement à celle de 1951-1969. Ainsi, on enregistre un déficit de 13\% (Banikoara), 11\% (Malanville), 18\% (Bembèrèkè), $12 \%$ (Kouandé) et $11 \%$ (Kandi). Le mois août, le plus arrosé a connu aussi une baisse générale de $14 \%$ (soit 40 $\mathrm{mm}$ ) de pluie. C'est d'ailleurs ce qui justifie le déficit hydrique observé; préjudiciable à la production agricole au centre et au nord du Bénin (Gnangle et al., 2011 ; Issa, 2012).

La fréquence des anomalies négatives entre 1970 et 1990 illustrée plus haut traduit la péjoration climatique qui a affecté toute la région (Houndénou, 1999; Vissin, 2007) comme le montre d'ailleurs la Figure 8. Mais, cette péjoration pluviométrique est plus marquée dans la région sous l'influence du climatique tropical humide (Kérou et Bembèrèkè, Banikoara) que sec (Kandi, Malanville), ce qui aura sans doute une influence dans la production agricole du milieu.

Les Figures 3, 6 et 8 confirment la baisse des précipitations et l'inégale répartition spatiale des précipitations dans le bassin. C'est ce qui détermine le développement des activités socioéconomiques et la vie des écosystèmes.

A cela s'ajoute la dégradation du milieu attribuée à la surexploitation des terres agricoles qui entraine une régression sensible des formations végétales naturelles et une 
progression des formations anthropiques (Vissin, 2007). Aujourd'hui, tous ces facteurs déterminent l'évolution de la production agricole et du dynamisme écologique dans le bassin.

\section{Evolution des unités paysagères dans le} bassin-versant béninois du fleuve Niger

De l'analyse de la Figure 9, il ressort que les formations végétales naturelles connaissent une régression sensible dans le bassin entre 1979 et 1992 . Ainsi, en 1992, les formations végétales vertes ont disparu pour laisser place aux formations végétales jaunes qui ne sont rien d'autre que des mosaïques de cultures et jachère. Les forêts denses sèches et galeries forestières sont passées de 328851 ha en 1979 à 3200 ha en 1992 soit une baisse de 99\%. Par contre, les agglomérations, les mosaïques de culture et jachère occupent respectivement une superficie de 22665 ha et 2215382 ha en 1992 contre respectivement 3971 ha et 945786 ha en 1979. Ceci corrobore les résultats Vissin (2007) qui ont montré l'anthropisation du bassin avec une forte dégradation des formations végétales (environ $70 \%$ ).

Cette forte anthropisation dans le bassin est facteur de dégradation des écosystèmes: fragilisation des terres, érodibilité des sols, pertes de la fertilité des sols suite au ruissellement, dégradation des eaux, ensablement du cours d'eau, eutrophisation.

\section{Impacts des changements environnementaux sur la production agricole}

L'accroissement démographique rapide et les effets nocifs des changements climatiques entraînent une augmentation de la demande en produits agricoles au Bénin, et dans le bassin-versant en particulier. Ainsi, l'utilisation des intrants agricoles ne se limite point à la production cotonnière mais aussi pour les produits vivriers (Vodounon, 2010).

Les impacts des changements climatiques (l'excès de pluies sur un temps court) sur le sol varient en fonction du niveau d'exploitation des écosystèmes. L'agressivité des pluies, ces dernières années, a contribué à l'intensification de l'érosion des sols, particulièrement des sols dénudés (Ahouansou, 2010). L'érosion aujourd'hui, marque tout le paysage et est devenu un sujet de grande préoccupation, surtout du fait du lessivage des engrais chimiques et $\mathrm{du}$ comblement des cours d'eau.

Cette pression des facteurs physiques et humains sur les sols engendre une dégradation des terres (Photo 1) et un fort transport des charges solides vers le lit des fleuves, une pollution des eaux et une apparition des maladies dites «mystiques» aujourd'hui selon les $97 \%$ des enquêtés. Avec cette dégradation de l'environnement, les sols s'appauvrissent davantage et l'utilisation des engrais s'accroît, avec une production agricole presque statique ou en baisse, même avec l'augmentation de la superficie emblavée (Figure 10).

L'analyse de la Figure 10 montre une très forte variabilité des rendements agricoles dans le bassin. La production agricole est en baisse par rapport à la superficie emblavée. Ce décalage est plus marqué pour la production du sorgho et de l'igname, qui constituent des produits alimentaires de première classe dans la région septentrionale du Bénin.

Avec l'introduction du maïs amélioré dans un environnement de déficit pluviométrique, le rendement n'est toujours pas reluisant. Ceci confirme le degré d'appauvrissement organique et minéral des terres agricoles, ce qui induit inévitablement des pertes progressives de récoltes très considérables selon $93 \%$ des enquêtés.

Signalons aussi qu'avec la dégradation de l'environnement (Photo 2), les cours d'eau, l'élevage et la pêche sont aussi impactés. Ainsi, les cours d'eau tarissent vite, les animaux ont de difficultés à s'abreuver. Cette destruction du couvert végétal constitue des menaces graves à l'environnement avec des possibilités de migration forcée des populations selon $75 \%$ des résultats issus des focus-groups.

Les changements climatiques ne sont pas sans conséquences sur le milieu et le quotidien des paysans. Sur le milieu, les 
conséquences se traduisent par de forte érosivité des sols, la prolifération de certaines espèces végétales (le striga par exemple), animales (les insectes ravageurs) et le tarissement précoce des mares et cours d'eau. Par contre, les effets des changements climatiques sur le quotidien des acteurs se traduisent par les baisses de rendements et les pertes de récolte. L'utilisation accrue des pesticides pour accroître les rendements est un facteur de risque environnemental avec une recrudescence de certaines maladies (la maladie de Gomboro, les diarrhées chez la volaille; la gale, la pneumonie, les troubles gastriques chez les ruminants) chez les animaux d'élevage et chez les paysans euxmêmes. Il s'agit de la prolifération du paludisme, des infections respiratoires (toux, rhume), l'anémie et des maladies diarrhéiques.

Le Tableau 1 présente la sensibilité des écosystèmes aux changements environnements dans le bassin. L'appréciation de ce degré de vulnérabilité des terres et eaux dans le bassin béninois a permis de mieux cerner les modes d'existence vulnérables aux aléas. En plus de l'analyse de la Figure 2 supra à la vulnérabilité biophysique aux aléas climatiques et environnementaux s'ajoute, l'analyse de la matrice de sensibilité.

Il ressort de l'analyse du Tableau I, une aggravation de la sensibilité et de la criticité des écosystèmes biophysiques du milieu aux changements climatiques et aux activités anthropiques sans cesse croissantes. Le bassin-versant béninois du fleuve Niger est sous la pression démographique (pratiques agricoles) et de la péjoration climatique avec des pertes potentielles de la biodiversité. A cet effet, les changements environnementaux globaux observés dans le bassin interpellent tous les acteurs à développer des stratégies d'adaptation face à l'intensification de la sécheresse, dégradation des terres et des eaux, pollution de l'écosystème.

\section{Stratégies d'adaptation des populations face aux changements environnementaux \\ La sécurité alimentaire est une préoccupation importante pour les communautés rurales qui dépendent de}

l'agriculture pluviale pour résoudre leurs problèmes de subsistance. A cet effet, les différentes stratégies d'adaptation développées par les populations pour résister aux effets induits par les changements climatiques, la dégradation du couvert végétal, des terres et la pollution des eaux varient suivant les secteurs d'activité (agriculture, élevage, pêche, exploitation forestière). Ainsi, l'adaptation aux changements climatiques est l'ensemble des initiatives prises par les populations pour garantir leur sécurité alimentaire et subvenir à leurs besoins quotidiens.

Dans le secteur agricole, nous pouvons citer: l'intensification de l'usage des fertilisants chimiques de synthèse; la lutte contre les ravageurs à travers l'usage des pesticides; les semis précoces sans labour avec sarclobuttage; les semis échelonnés et/ou semis répétés; l'extension des terres agricoles; la réduction des superficies consacrées à la culture du coton ; la culture de contre-saison dans les bas-fonds; l'utilisation de nouvelles variétés de culture/semences améliorées; l'abandon progressif du calendrier agricole ancien.

Concernant l'élevage, les stratégies développées par les populations pour s'adapter aux crises que provoquent les changements climatiques sont: l'utilisation des fanes de légumineuses pour l'alimentation du bétail; baignade des animaux dans les cours d'eau; partage de l'eau de consommation avec le bétail, la pénétration des animaux dans les aires protégées (Parc W), mais défendue par les lois, car cela cause d'autres dommage à la biodiversité de ce milieu.

Pour la pêche, les stratégies développées sont : la mise en défens des mares et autres points d'eau; le surcreusement des mares; l'utilisation des engins de pêche appropriés et recommandés; l'interdiction de l'usage des pesticides dans les cours d'eau au profit des plantes venimeuses. 
Tableau 1 : Appréciation du degré de vulnérabilité des écosystèmes biophysiques (eaux et terres).

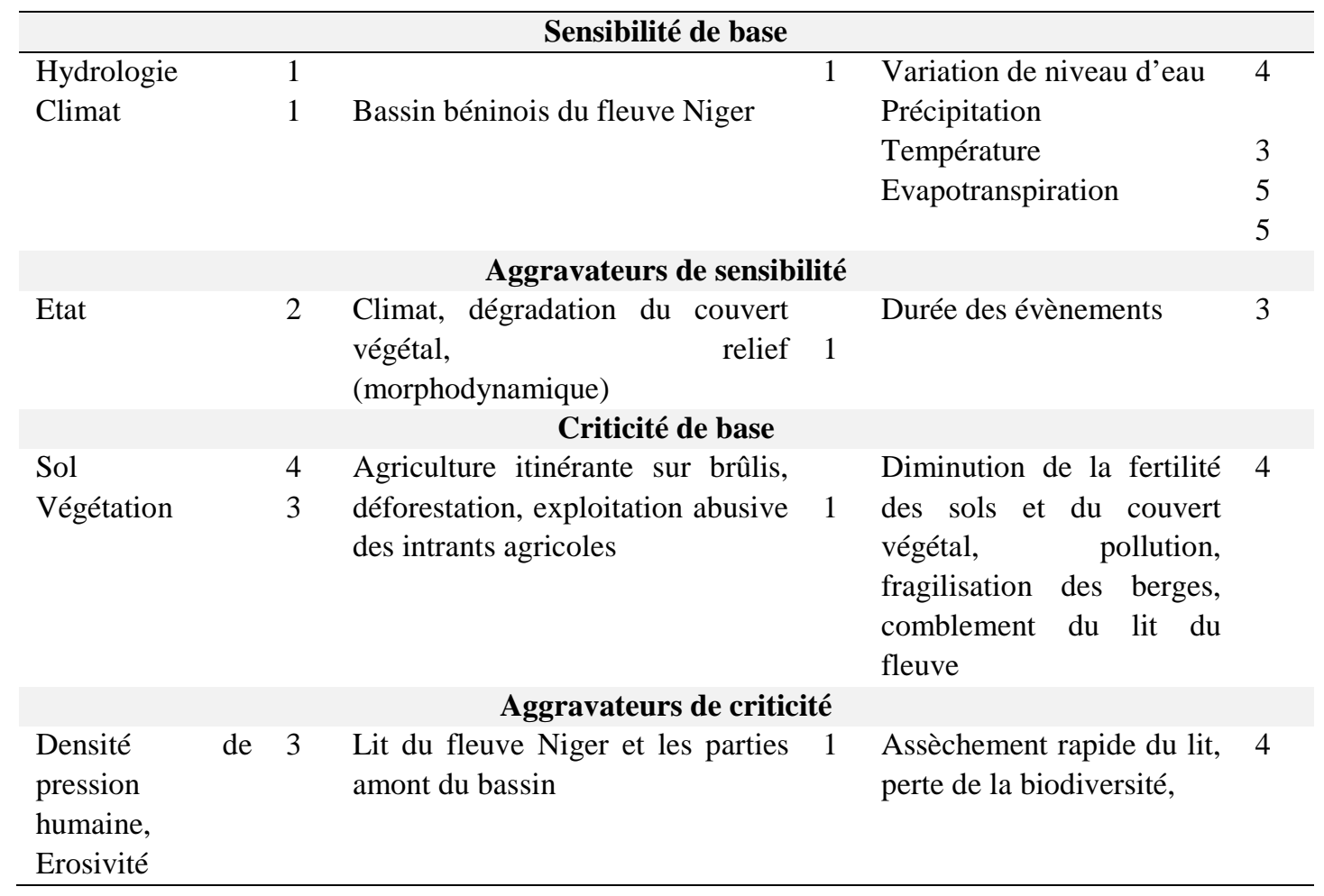

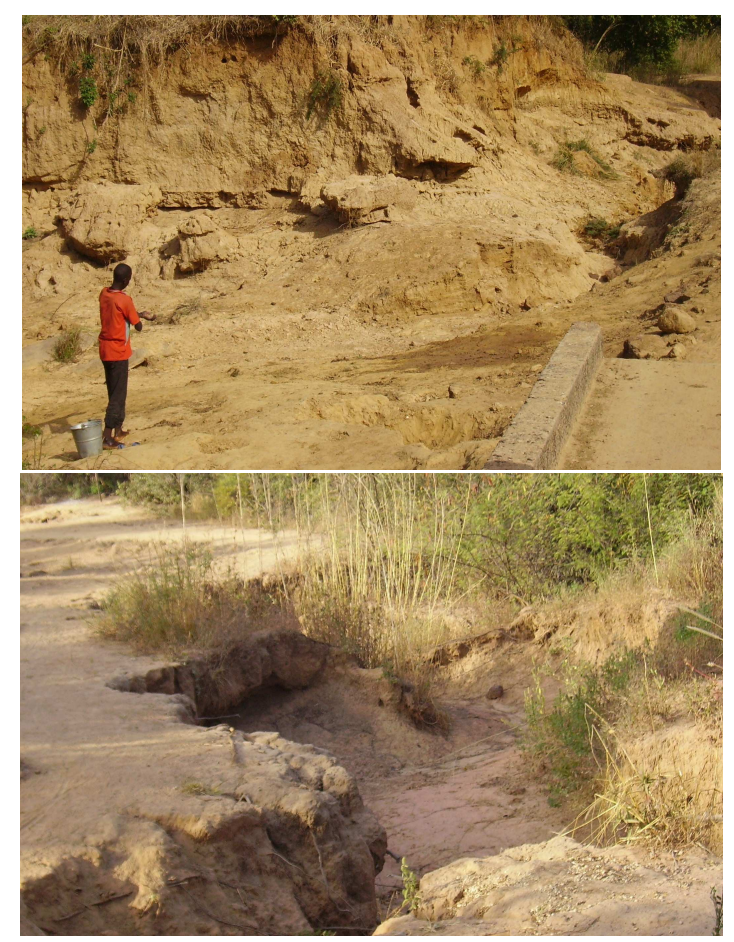

Photo 1: Dégradation des terres et érodibilité du sol dans le bassin béninois du fleuve Niger. Cliché Vissin, 2006. 

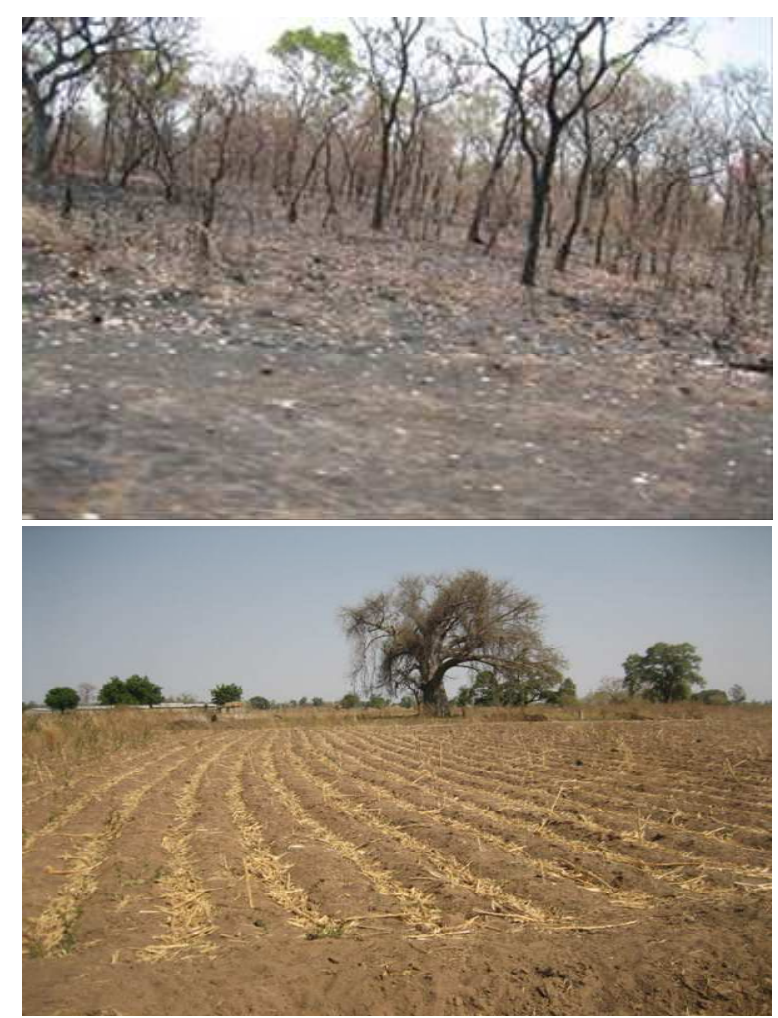

Clichés LACEEDE, 2003 et Amoussou, 2007.

Photo 2 : Dégradation du couvert végétal dans le bassin-versant suite aux pratiques agricoles non durable.

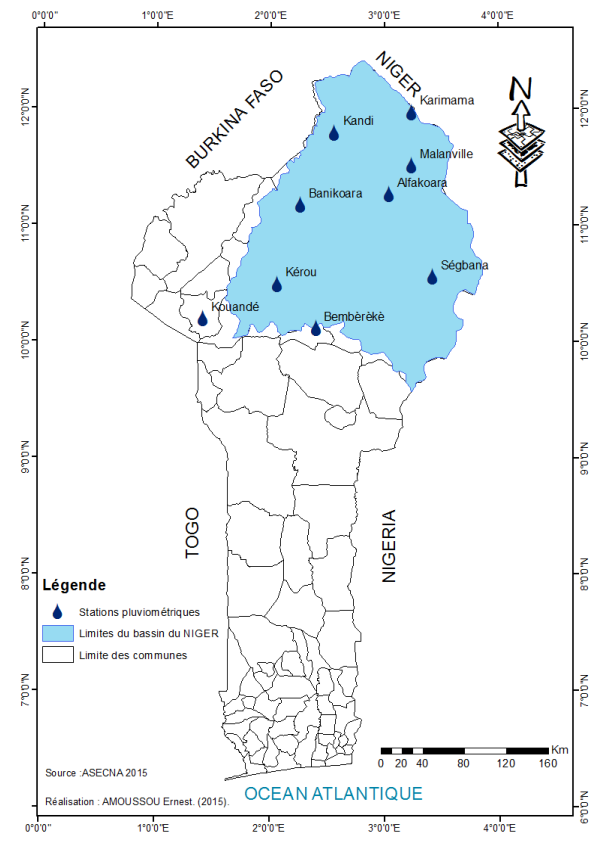

Figure 1: Localisation du bassin béninois du fleuve Niger. 


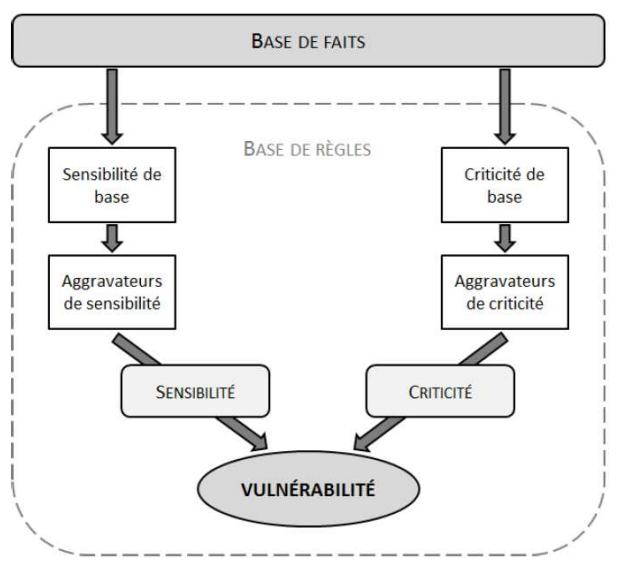

Figure 2 : Méthodologie sur la vulnérabilité.

NB : Le barème d'évaluation de l'ampleur de la vulnérabilité des terres et des eaux est noté de 1 à $5: 1=$ faible, $2=$ Assez faible ; $3=$ Moyen $; 4=$ Assez fort (aggravation) et $5=$ fort (aggravation).

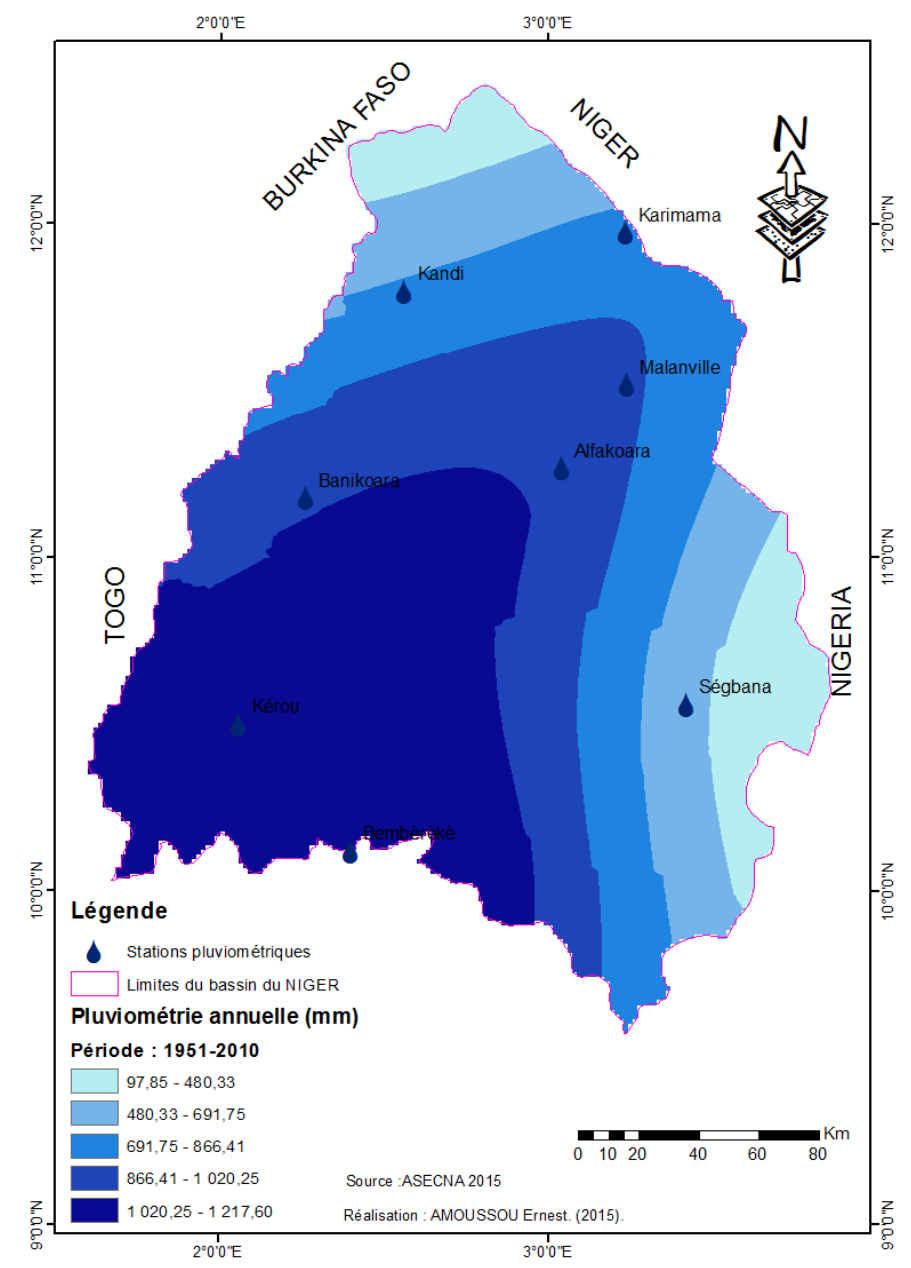

Figure 3 : Evolution spatiale des précipitations dans le bassin béninois du fleuve Niger. 


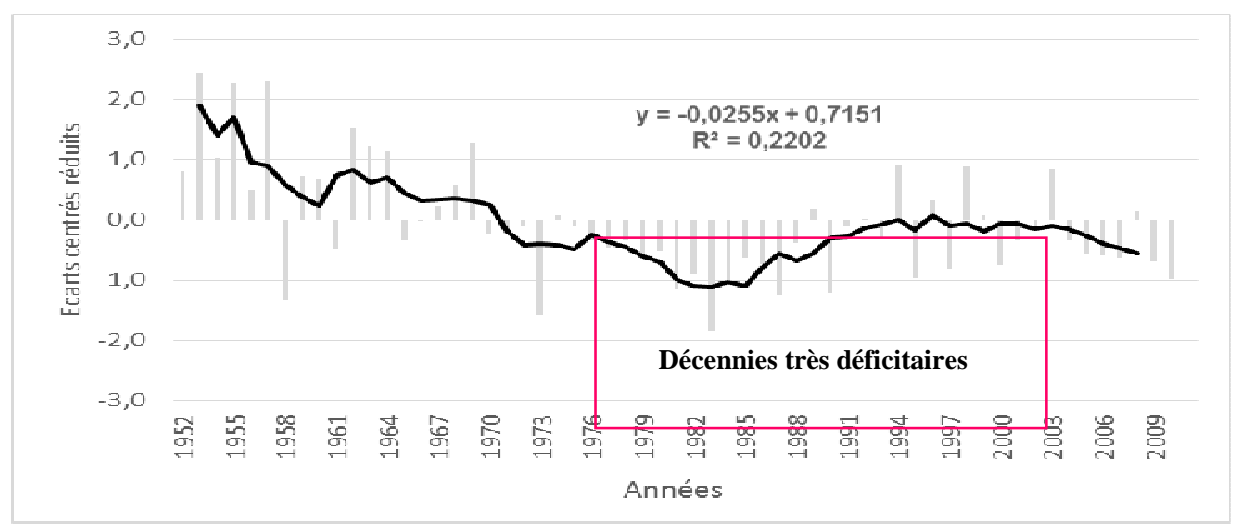

Figure 4: Variabilité interannuelle de la pluie de certaines stations du bassin béninois du fleuve Niger de 1951 à 2010.

NB : Les barres représentent les anomalies de la pluie, la courbe noire représente la moyenne mobile sur 5 ans.

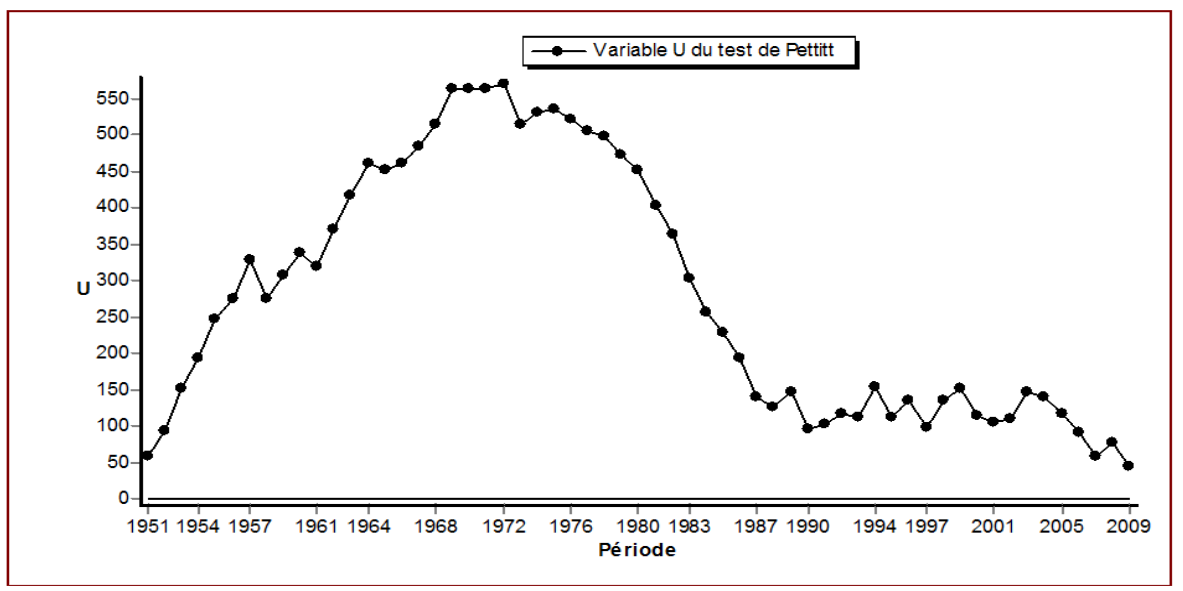

Figure 5: Évolution de U variable d'essai de Pettitt le bassin béninois du fleuve Niger.

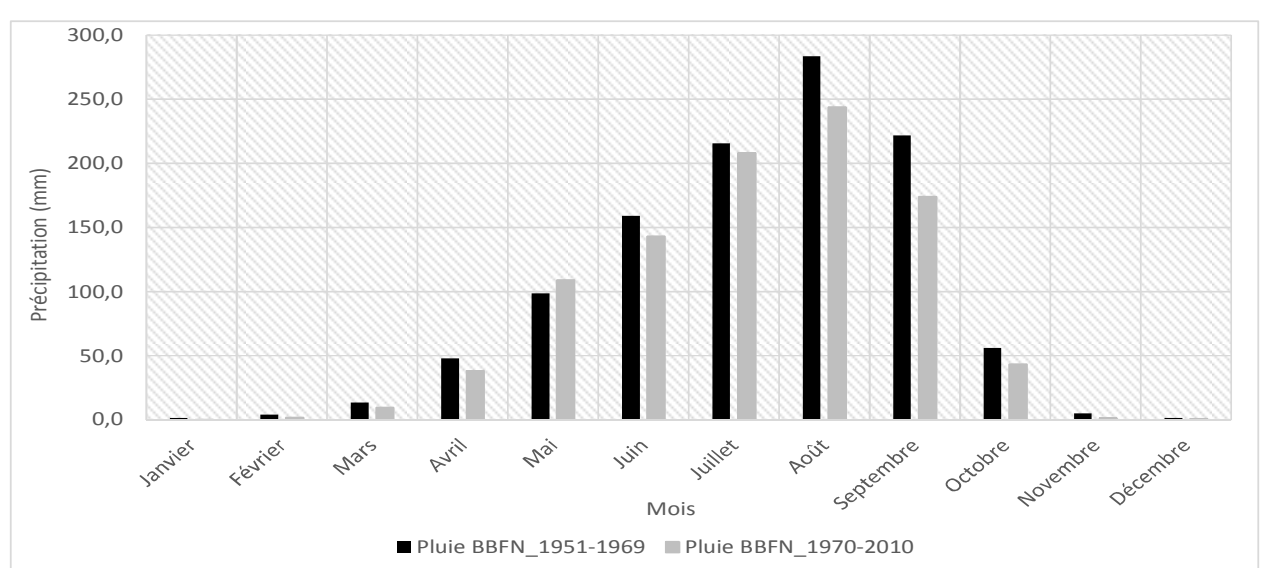

Figure 6 : Variation des précipitations des sous-périodes 1951-1969 et 1970-2010 du bassin du fleuve Niger. 

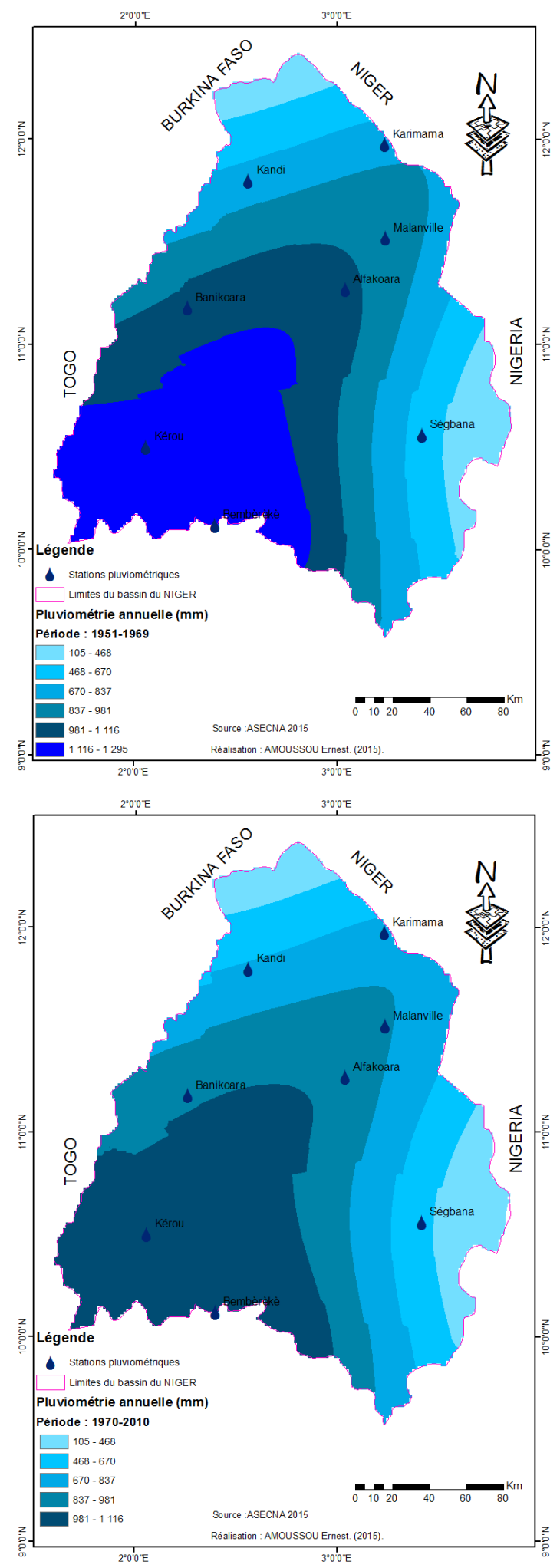

Figure 7 : Evolution spatiale des précipitations suivant les deux sous-périodes dans le bassin béninois du fleuve Niger. 


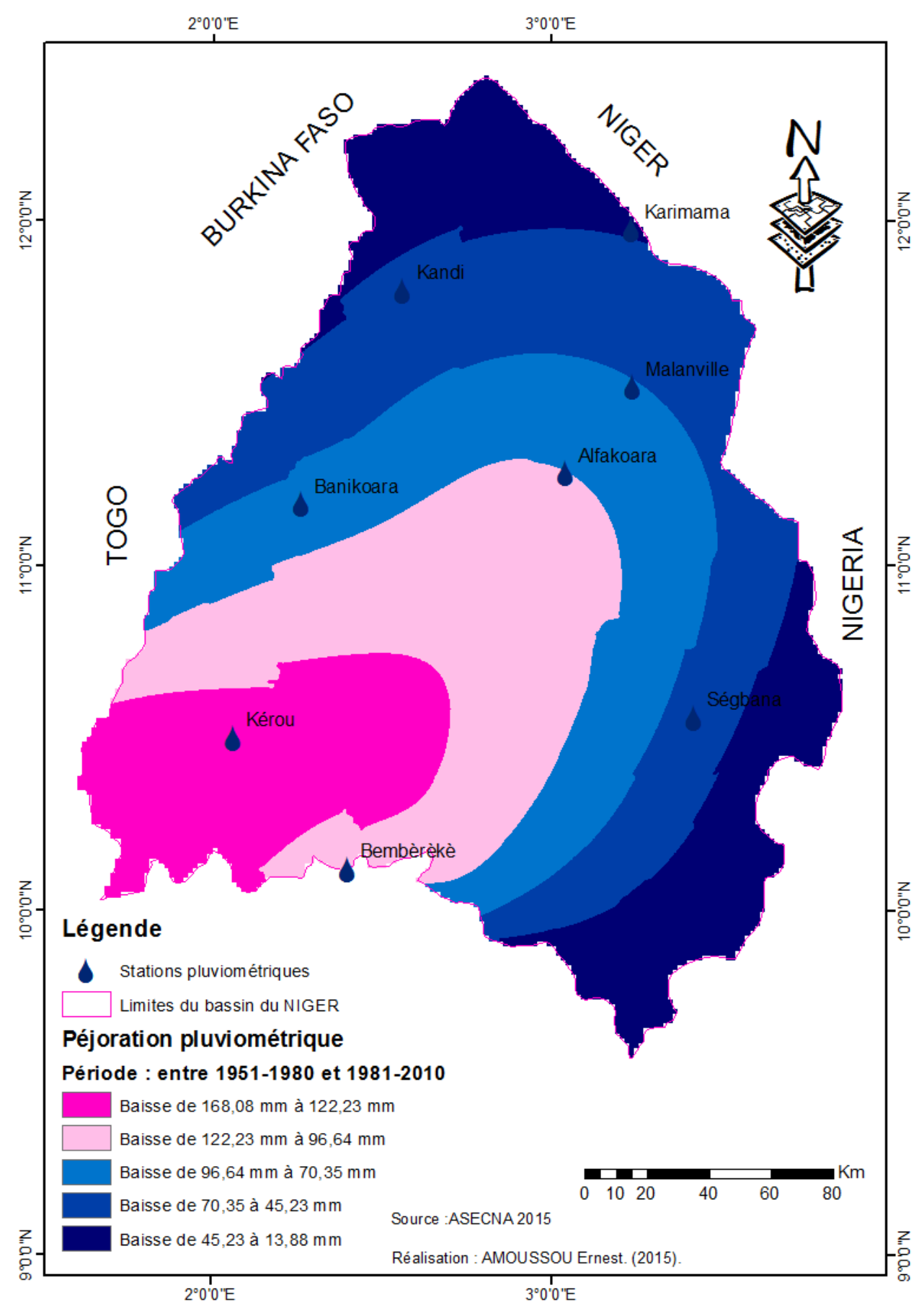

Figure 8 : Péjoration pluviométrique dans le bassin entre les deux sous-périodes. 


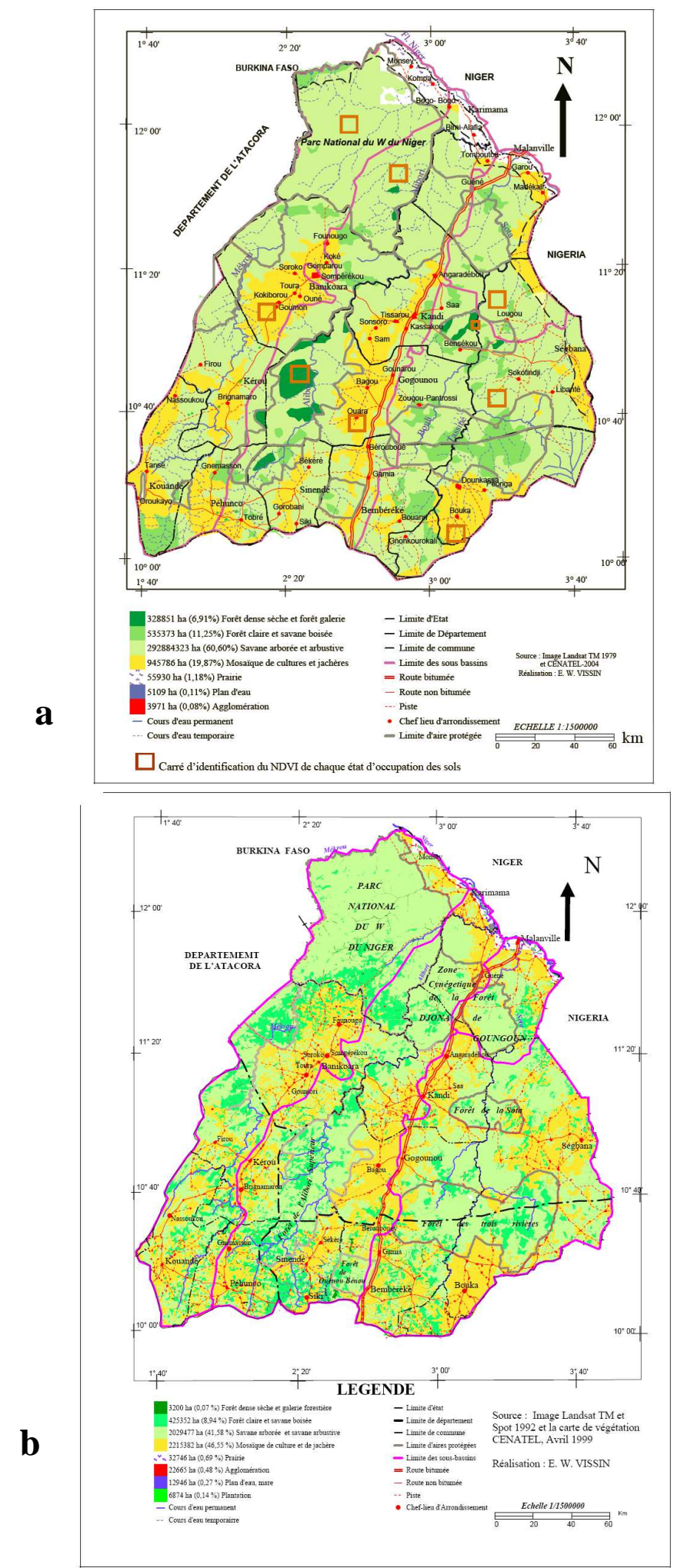

Figure 9 : Etats d'occupation des sols du bassin béninois du fleuve Niger en 1979 (a) et 1992 (b).

Source : Vissin, 2007. 


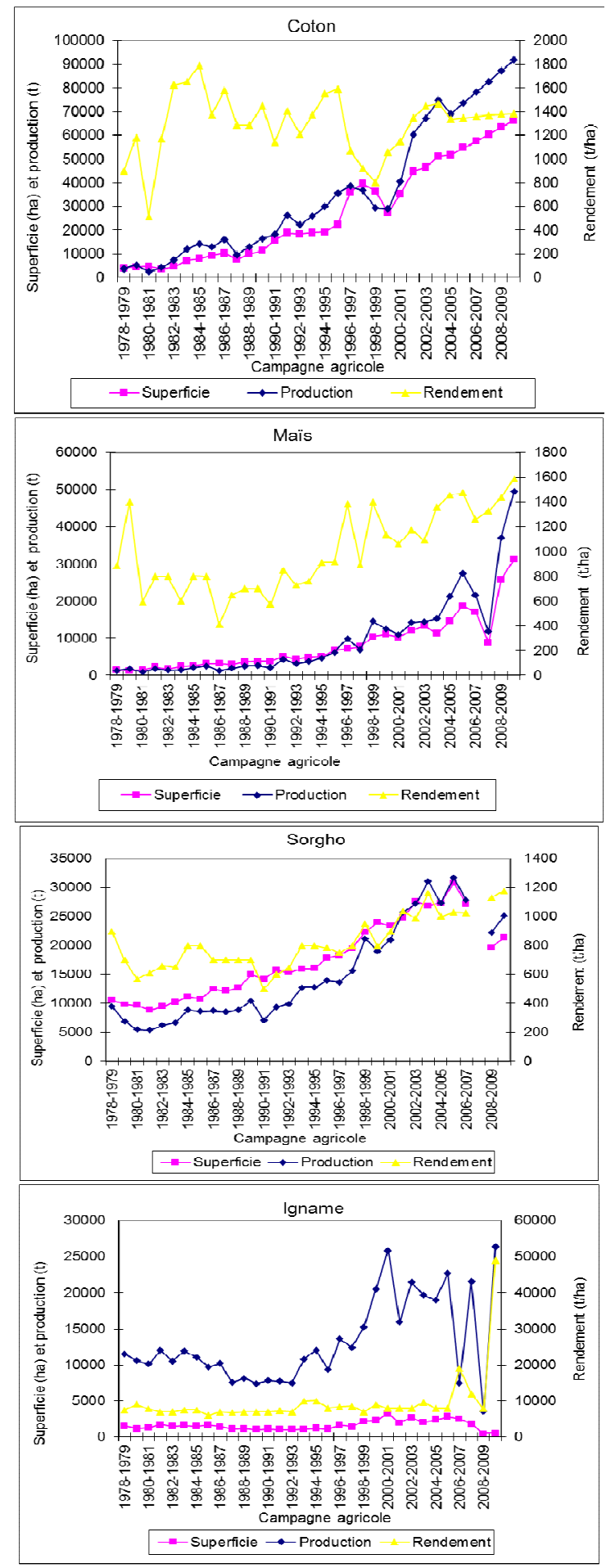

Figure 10: Evolution de la surface d'emblavure et la production de coton et les produits alimentaires de 1978-2010 à Banikoara.

(Source: MAEP, 2013) 


\section{DISCUSSION}

Les résultats obtenus s'accordent sur l'occurrence de fortes fluctuations interannuelles des précipitations avec une tendance à la baisse pour toutes les stations du bassin. Ils montrent la succession de périodes sèches et de périodes humides.

L'analyse de l'évolution interannuelle des précipitations dans le bassin béninois du fleuve Niger met en évidence trois phases: une phase très humide, une phase sèche et une phase humide. Ces résultats corroborent avec ceux obtenus par Vissin (2007), Ahouansou (2010) et Vodounon (2010) dans le bassin et ceux obtenus aussi dans le bassin sédimentaire côtier par Amoussou (2010) et Totin (2010) de 1961 à 2000. Ils sont aussi confirmés par Ozer et al. (2009) et Badjana et al. (2014) en Afrique de l'Ouest. En dépit de cette baisse générale, les épisodes secs annuels succèdent les épisodes plus humides, sans pour autant qu'aucun caractère cyclique n'apparaisse (Vissin, 2007). Une étude de la recherche de rupture stationnarité a montré une modification de l'évolution des pluies autour des années 1970 comme l'ont signalé plusieurs chercheurs au Bénin (Houndénou, 1999 ; Vissin, 2007 ; Ozer et al., 2009) et en Afrique de l'Ouest (Servat et al., 1999 ;Hubert et al., 1998 ; Niel et al., 2005 ; Bajana et al., 2014).

Cette péjoration climatique associée à la dégradation des formations naturelles au profit des formations anthropiques (Vissin, 2007) ne sont pas sans conséquence sur la production agricole. Cette fragilisation du couvert végétal a engendré une dégradation des terres et pour corollaire une perte de fertilité dans les terres agricoles comme l'avaient déjà signalé Vissin (2007) ; Adam soulé et al. (2008); Ahouansou (2010); Vodounon (2010); Akouèhou et al. (2013). De plus, la baisse des rendements agricoles, des écarts très marqués entre superficie emblavée et production agricole sont attribués aux problèmes environnementaux notamment à la dégradation du couvert végétal, à l'érosion hydrique, à la pollution des terres et à la dégradation du régime pluviométrique.
Ces résultats corroborent avec ceux de Amoussou et al. (2006), Tschakert (2007), Adam et al. (2008), Abou et Djambouto (2009), Mertz et al. (2009), Ozer et al. (2009), Gnangle et al. (2011), Katé et al. (2016) en ce sens que pour eux, la diminution de rendements des cultures est due à des changements climatiques et à la forte pression sur les terres agricoles. Une telle situation pourrait engendrer une insécurité alimentaire. Dans cette situation le développement d'un plan stratégique d'adaptation pouvant permettre de faire face aux problèmes de dégradation des terres et des eaux dans un contexte de changements climatiques est nécessaire.

\section{Conclusion}

$\mathrm{Au}$ terme de cette étude, il revient de retenir une diminution des lames d'eau précipitées des années 1970 à nos jours de plus de $12 \%$ malgré la légère reprise des années 1990, mais inégalement répartie. Cette baisse pluviométrique ajoutée aux pratiques agricoles non durable a entraîné une baisse des rendements agricoles avec pour corollaire une baisse de revenu des populations, une baisse du niveau d'eau dans les différents sous-bassins du bassin béninoise du fleuve Niger. Ceci a conduit à une utilisation intensive des intrants agricoles qui a amplifié la pollution des eaux et amenuisement progressif des terres.

Dans cette situation de plus en plus aggravée, des stratégies sont envisagées: le reboisement en amont des retenues d'eau, la diversification des sources de revenues avec les ressources endogènes, la gestion de l'eau à travers la mise en défens et le surcreusement des mares et points d'eau. Quelques mesures de remède telles que l'utilisation de la partie organique des ordures, les bouses des vaches pour restaurer les sols; l'épandage des matières organiques dans les champs sont conseillées. Il a été proposé de sauvegarder 10 arbres (néré, karité,...) par hectare dans les champs de coton. Mais, il ne suffit pas de constater que les populations ont mis au point 
des stratégies d'adaptation. Au-delà de ce constat, il se pose plusieurs questions:

- Si ces stratégies découlent bien évidemment, du vécu des populations, elles induisent de facto l'idée de cycle et non plus de tendance. Quelles en seraient alors les causes et les périodes : éruptions solaires ?, oscillation quasi biennale ?, cycle de Milankovitch ? On est de toute évidence loin de la thèse de changements climatiques (pourtant irréfutable), mais simplement dans la variabilité climatique, avec des cycles et des périodes qui s'entremêlent ou se chevauchent.

- On peut aussi penser que ces stratégies ont été mises en place dans un autre contexte climatique ou dans une autre zone climatique (le Sahara en cours d'aridification par exemple) et transférées en milieu soudanien voire subéquatorial lors des migrations de population. Mais alors, quelles seraient leurs limites d'efficacité si les tendances actuelles persistent au cours des décennies à venir?

Des pistes d'investigations restent ouvertes par des méthodes d'analyse de prospective sociale et statistiques.

\section{CONFLIT D'INTÉRÊT}

Pas de conflit d'intérêt.

\section{CONTRIBUTIONS DES AUTEURS}

EA s'est occupé de la spatialisation des données, analyse et rédaction de l'article; SHTV a fait le traitement statistique et cartographique des données climatologiques et l'interprétation; $\mathrm{AH}$ a fourni les données sur les productions agricoles et leur traitement; EWV a réalisé les cartes d'occupation du sol dans le bassin béninois du fleuve niger et a fait l'analyse ; $\mathrm{CH}$ a analysé les données agricoles ; GM s'est occupé de la correction et de la définition de l'approche sur la vulnérabilité ; MB a contribué à la lecture et à l'application de théorie des changements climatiques.

\section{REFERENCES}

Abdou Y, Djambouto B. 2009. Les justifications et les faisabilités économiques et sociale du traitement des dunes de sable dans le Département de Gouré au Niger : cas du village de Tchago. Geo. Eco. Trop., 33: 125 - 132.

Adam Soulé I, Totin VSH, Amoussou E. 2008. Systèmes culturaux et dégradation de l'environnement dans la ceinture cotonnière de Banikoara (Bénin). Revue Science Environnement, 004 : 41-58.

Ahouansou M. 2010. Influence de la dynamique du couvert végétal et du changement climatique sur les ressources en eau dans le bassin de la Mékrou à l'exutoire de Kompongou à l'horizon 2025. Mémoire de formation Master II (Ex-DESS), CIFRED, Université d'Abomey-Calavi, 135 pages.

Akouehou GS, Houndonougbo A, Tenté B. 2013. La dynamique des systèmes de production dans les terroirs agricoles riverains de la forêt intercommunale de Fita-Agbado dans les communes de Dassa-Zoumé et de Savalou, Département des Collines au Centre du Bénin. Int. J. Biol. Chem. Sci., 7(5): 1877-1891.

Amoussou E. 2010. Variabilité pluviométrique et dynamique hydrosédimentaire du bassin-versant du complexe fluvio-lagunaire Mono-AhéméCouffo (Afrique de l'Ouest). Thèse de Doctorat unique, Université de Bourgogne, Dijon.

Amoussou E, Oyédé LM, Boko M. 2006. Variabilité pluvio-hydrologique et production agricole dans le bassin du Couffo au Bénin (Afrique de l'ouest). In «Risques liés au temps et au climat ». Acte de Colloque AIC, Epernay, France ; 67-72.

Badjana HM, Hounkpè K, Wala K, Batawila K, kpagana K, Edjamé KS. 2014. Analyse de la variabilité temporelle et spatiale des séries climatiques du Nord du Togo entre 1960 et 2010. European Scientific Journal, 10(11): 257-275.

Badolo M. 2009. Cahier des changements climatiques. Bulletin Mensuel d'Information sur les Changements Climatiques de l'Institut d'Applications et de Vulgarisation en Science. 
Gbaguidi AA, Faouziath S, Orobiyi A, Dansi M., Akouegninou B A., Dansi A. 2015. Connaissances endogènes et perceptions paysannes de l'impact des changements climatiques sur la production et la diversité du niébé (Vigna unguiculata (L.) Walp.) et du voandzou (Vigna subterranea (L) Verdc.) au Bénin. Int. J. Biol. Chem. Sci., 9(5): 2520-2541.

GIEC. 2007. Bilan des changements climatiques. Contribution des Groupes de travail I, II et III au quatrième Rapport d'évaluation du Groupe d'experts intergouvernemental sur l'évolution du climat [Équipe de rédaction principale, Pachauri, R.K. et Reisinger, A.]. GIEC, Genève, 103 p.

Gnangle PC, Egah J, Baco MN, Gbemavo CSJ, Kakaï RG, Sokpon N. 2015. Perceptions locales du changement climatique et mesures d'adaptation dans la gestion des parcs à karité au NordBénin. Int. J. Biol. Chem. Sci., 6(1): 136149.

Gnanglè PC, Glèlè Kakaï RL, Assogbadjo AE, Vodounon S, Yabi JA, Sokpon N. 2011. Tendances climatiques passées, modélisation, perceptions et adaptations locales au Bénin. Climatologie, 8: 26-40.

Gorokhovich Y, Voustianouk A. 2006. Accuracy assessment of the SRTM-based elevation data by CGIAR using field data from USA and Thailand and its relation to the terrain characteristics. Remote Sensing of Environment, 104(4): 409-415.

Houndénou C. 1999. Variabilité climatique et maïsiculture en milieu tropical humide : L'exemple du Bénin, diagnostic et modélisation. Thèse de Doctorat, Université de Bourgogne, Dijon, 390 p.

Hubert P, Servat E, Pature J-E, Kouam B, Bendjoudi H, Carbonnel J-P, Lubès-Niel H. 1998. La procédure de segmentation, dix ans après. IAHS Publication, 252: 267-273.

Issa SM. 2012. Changements climatiques et agrosystèmes dans le moyen Bénin : impacts et stratégies d'adaptation. Thèse de Doctorat Unique, Université d'Abomey-Calavi, 273p.
Katé S, Azontondé AH, Dagbenonbakin GD, Sinsin B. 2016. Effets des changements climatiques et des modes de gestion sur la fertilité des sols dans la commune de Banikoara au nord-ouest du Benin. Int. J. Biol. Chem. Sci., 10(1): 120-133.

Mertz O, Mbow C, Reenberg A, Diouf A. 2009. Farmers' Perceptions of Climate Change and Agricultural Adaptation Strategies in Rural Sahel. Environmental Management, 43: 804-816.

Niel H, Leduc C, Dieulin C. 2005. Caractérisation de la variabilité spatiale et temporelle des précipitations annuelles sur le bassin du lac Tchad au cours du 20ème siècle. Hydrological Sciences Journal, 50: 221-243.

Oettli P. 2008. Précipitations et relief en Afrique orientale et australe: Modélisations statistiques et géostatistiques. Thèse de Doctorat à l'Université de Bourgogne, Centre de Recherches de Climatologie. France, 272 $\mathrm{p}+$ Annexes.

Oettli P, Camberlin P. 2005. Influence of topography on monthly rainfall distribution over East Africa. Climate Research, 28: 199-212.

Olivry F. 2012. Exploration d'une méthode d'évaluation de la vulnérabilité des systèmes essentiels d'une région face aux extrêmes météorologiques dans un contexte de changements climatiques. Mémoire présenté en vue de l'obtention du diplôme de maîtrise ès sciences appliquées (Génie industriel), Université de Montréal, Ecole polytechnique de Montréal, $144 \mathrm{p}$.

Ouorou Barrè I. 2014. Contraintes climatiques, pédologiques et production agricole dans l'Atacora (Nord-Ouest du Bénin). Thèse de Doctorat Unique, Université d'Abomey-Calavi, 229 pages.

Ozer P, Hountondji YC, Laminou Manzo O. 2009. Evolution des caractéristiques pluviométriques dans l'est du Niger de 1940 à 2007. Geo. Eco. Trop., 33: 11-30.

Philippon N. 2002. Une nouvelle approche pour la prévision statistique des précipitations saisonnières en Afrique de 
l'Ouest et de l'Est : méthodes, diagnostics (1968-1998) et applications (2000-2001).

Thèse de Doctorat Université de Bourgogne, CRC - CNRS UMR5080, $241 \mathrm{p}$.

Servat E, Paturel J-E, Lubes-Niel H, Kouame B, Masson JM, Travaglio M, Marieu B. 1999. De différents aspects de la variabilité de la pluviométrie en Afrique de l'ouest et centrale non sahélienne. Revue des Sciences de l'Eau, 12(2): 363387.

Totin VSH, Clédjo P, Afouda A, Boko M. 2009. Variabilité pluviométrique et bilan climatique dans le bassin de la Volta au Bénin. Climat et Développement, 8: 8194.

Totin VSH. 2010. Sensibilité des eaux souterraines du bassin sédimentaire côtier du Bénin à l'évolution du climat et aux modes d'exploitation : Stratégies de gestion durable. Thèse de Doctorat,
Université d'Abomey-Calavi, Cotonou, Bénin, 283 p.

Tschakert P. 2007. Views from the vulnerable: Understanding climatic and other stressors in the Sahel. Global Environmental Change, 17: 381-396.

Vissin EW. 2007. Impact de la variabilité climatique et de la dynamique des états de surface sur les écoulements du bassin béninois du fleuve Niger. Thèse de doctorat, Université de Bourgogne, Dijon, $311 \mathrm{p}$.

Vodounon AJ. 2010. Impacts des systèmes culturaux sur la biodiversité, les états de surface et les écoulements dans le bassinversant de la Sota. Mémoire de formation Master II (Ex-DESS), CIFRED, Université d'Abomey-Calavi, 95 p.

Yabi I, Afouda F. 2012. Extreme rainfall years in Benin (West Africa). Quaternary International, 262(7): 39-43. 